\title{
Paraxially annihilating vortex-beams in uniaxial crystals
}

\author{
${ }^{1}$ Fadeyeva T., ${ }^{2}$ Valkov I. and ${ }^{1}$ Volyar A. \\ ${ }^{1}$ Physics Department, Taurida National V.I. Vernadsky University, 4 Vernadsky \\ Ave., 95007 Simferopol, Ukraine, e-mail: tatyana.fadeyeva@gmail.com \\ ${ }^{2}$ Department EKSA, Technikal University-Sofia, Plovdiv Branch, 25 Tsanko \\ Diustabanov St., 4000 Plovdiv, Bulgaria
}

Received: 29.12.2009

\begin{abstract}
Unique properties of paraxially annihilating beams have been considered. We have derived the expressions for this beam type obeying the Maxwell's equations. It has been revealed that the beams originate from mismatching of the electric and magnetic fields and the evanescent waves in the field components. When the electric and magnetic fields become totally matched in the paraxial approximation, the paraxially annihilating beams vanish.
\end{abstract}

Keywords: nonparaxial beam, optical vortex, uniaxial birefringent crystal

PACS: 41.85.-p, 42.25.Ja, 42.25.Lc

UDC: $535.3,535.5$

\section{Introduction}

There is a common opinion in optics that basic distinction between paraxial and nonparaxial beams is that the characteristic angle $\alpha$ of divergence (or convergence) of the paraxial beams must be very small so that $\sin \alpha \approx \alpha$ and $\cos \alpha \approx 1-\alpha^{2} / 2$, whereas the nonparaxial beams are characterised by arbitrarily large real values of that angle. In other words, simple decrease in the characteristic angle $\alpha$ transfers the beam from the nonparaxial region into the paraxial one. However, any general analysis of this point for both the scalar and vector representations of the light fields, even for a free space or a homogeneous medium, demonstrates that this is not the case.

Indeed, this oversimplified approach is based, as a rule, on representation of light beams in the form of plane-wave superpositions. On the other hand, it is well known that homogeneous plane waves propagating in some medium cannot, in principle, form a complete basis for representation of general solutions to the wave equation [1]. Apparent incompleteness of such a wave set can be easily seen on the example concerned with representation of spectral integral as a solution to the scalar wave equation. For the monochromatic waves with the frequency $\omega$ we have

$$
\left(\nabla^{2}+k^{2} n^{2}\right) \Psi(x, y, z)=0,
$$

where $k=\omega / c=2 \pi / \lambda$ stands for the wave number, $\lambda$ and $c$ respectively the free- 
space wavelength and light velocity, and $n$ the refractive index of a given medium. The solution to Eq. (1) may be written for axially symmetric field distribution with the aid of the following set of plane waves [2]:

$$
\Psi_{m}(r, \varphi, z)=\pi e^{i m \varphi} \int_{0}^{\infty} d k_{\perp} U\left(k_{\perp}, z=0\right) J_{m}\left(k_{\perp} r\right) \exp \left[i z \sqrt{k^{2}-k_{\perp}^{2}}\right],
$$

where $U\left(k_{\perp}, z=0\right)$ is the field distribution in the space of wave vectors $\left(k_{x}, k_{y}, k_{z}\right)$, $J_{m}(x)$ the Bessel function of the first kind, $k_{\perp}=\sqrt{k_{x}^{2}+k_{y}^{2}}$, and the index $m=0,1,2, \ldots$ in the function $\Psi_{m}$ specifies the topological charge of vortex.

A set of uniform waves is given by the region $0 \leq k_{\perp} \leq k$. These waves propagate through a medium without changing their amplitudes. Their propagation directions lie inside the angular region $-\pi \leq \alpha \leq \pi$, forming a homogeneous field distribution $\Psi_{\text {hom }}(r, \varphi, z)$. Nonetheless, in order to form the wave field as a whole, it is also necessary to take into account the second set of waves with the transverse wave numbers inside the angular region $k<k_{\perp}<\infty$, which contribute to the field $\Psi_{e v}(r, \varphi, z)$, so that the total field is given by the superposition

$$
\Psi=\Psi_{\text {hom }}(r, \varphi, z)+\Psi_{e v}(r, \varphi, z) .
$$

The properties of such a peculiar field differ radically from those of the uniform waves. First of all, their field amplitudes are exponentially reduced to zero along the $z$ direction. Second, they oscillate along the direction $\operatorname{Re} \alpha$ with the wavelength of $\lambda_{e v} \geq 2 \pi /[k \cosh (\operatorname{Im} \alpha)]$, which is smaller than that for the homogeneous plane waves ( $\lambda_{\text {hom }} \leq 2 \pi / k$ [1]). These wave fields have come to be called as evanescent waves [3-5]. They cannot be represented in terms of ordinary plane waves, except for some special cases [1]. The evanescent waves exist not only in (or near) inhomogeneous media but are inherent to side lobes of nonparaxial Gaussian beams propagating in a free space or a homogeneous medium $[1,6]$, i.e. the waves containing phase singularities with alternated topological charges. Thus, the evanescent waves contribute essentially to the field structure and so any simple manipulations with the plane waves cannot permit describing all the properties of nonparaxial beams, in contrast to their paraxial analogues.

The major objection to the above consideration is that the evanescent waves do not contribute to the far field [7]. This should mean that description of the near field needs taking these waves into account, though we can restrict ourselves to a simple superposition of plane homogeneous waves when characterising the singular structure of the far field of both the paraxial and nonparaxial beams. However, this approach makes a sense when applied to the scalar fields only. The nonparaxial light beams are vector-like objects characterised additionally by their magnetic fields. In general case, the singular structures of the electric and magnetic fields do not coincide, even if the contribution of evanescent

Ukr. J. Phys. Opt. 2010, V11, №2 
waves is very small [8]. The singular lines of these fields can be matched with each other only in the paraxial approximation. The topological structure of such complex singularities needs special approaches, e.g., in the framework of Riemann-Silberstein vortices [9, 10] or the Poynting vector singularities [11-14] describing common polarisation singularities for both the electric and magnetic fields. Naturally, a spectrum of singular properties of the nonparaxial beams is sufficiently richer than that for the paraxial beams. When the beam waist radius increases (i.e., when the beam tends to become paraxial), some unique properties of that beam vanish. This process is very often accompanied by annihilation of the light beam on its own.

In the present work, we consider one of the examples of nonparaxial vortex-beams propagating along the optic axis of optically uniaxial crystal. It annihilates in the process of paraxial transferring and that is why we call it as a 'paraxially annihilating vortexbeam'.

\section{On the boundary problem for crystals}

As a rule, the evanescent waves are associated with boundary waves arising at a boundary face of adjacent media due to total internal reflection of a plane wave [3], although these waves can also exist, as mentioned above, in nonparaxial beams propagating in a homogeneous medium. Nonparaxial beams in crystals represent the brightest example of the case when the evanescent waves manifest themselves, on the one hand, as a constituent of nonparaxial beams in a homogeneous optically birefringent medium and, on the other hand, as waves matching the beam in an isotropic medium and the eigenmode beam in a crystal at its input face.

It is worth noting that the problem of matching the beam fields in the isotropic and anisotropic media is one of the most important points in the modern optics [15]. Its complexity might be judged by the fact that, to the authors' knowledge, the beam matching problem has not yet been solved in the explicit form even for relatively simple case of interface of two planar homogeneous isotropic media [16, 17]. The approach for beam matching at the interface of a homogeneous medium and a crystal is based on the principles suggested in the works $[16,17]$. It is restricted to consideration of either a paraxial approximation [18] or a far radiation field [19]. At the same time, the major difficulties of the problem refer to a correct form of transmission and reflecting coefficients rather than representation of the field inside the crystal near the interface. Following the approach [19], we will suppose that the electromagnetic field is known at the boundary, disregarding the problem of field matching at the interface. To begin with, we will analyse the main physical processes responsible for matching of the fields inside a homogeneous medium and an optically uniaxial crystal.

Let the field in a homogeneous isotropic medium at a crystal interface correspond to the nonparaxial beam containing both homogeneous and evanescent waves, while the refractive indices of the crystal be larger than that of the isotropic medium $\left(n_{\text {medium }}<n_{o}, n_{e}\right)$. The eigenmode beams in the crystal also contain both types of the 
waves. However, homogeneous waves of the isotropic medium will excite in the crystal only a set of homogeneous waves (see Fig. 1). In the isotropic medium with $k_{\perp}=k_{\text {medium }}$ the latter waves propagate along the crystal interface, forming a standing wave. The similar boundary wave in the crystal is a result of superposition of homogeneous ordinary and extraordinary waves propagating in the direction opposite to that of the beam in the isotropic medium, so that we have $k_{\perp}^{(o, e)}<k_{c r y s t a l}^{(o, e)}$. They form a set of homogeneous waves in the crystal responsible for matching with the boundary wave in the isotropic medium. It should be noticed that the ordinary and extraordinary waves have different angular spectra because of their different propagation constants, $k_{o}$ and $k_{e}$.

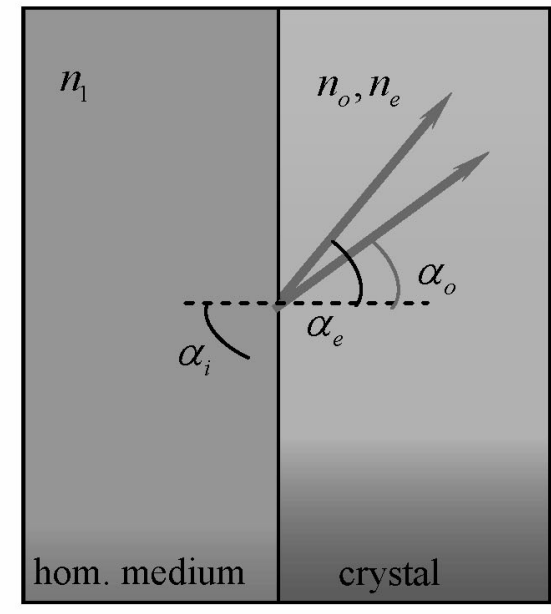

homogeneous waves

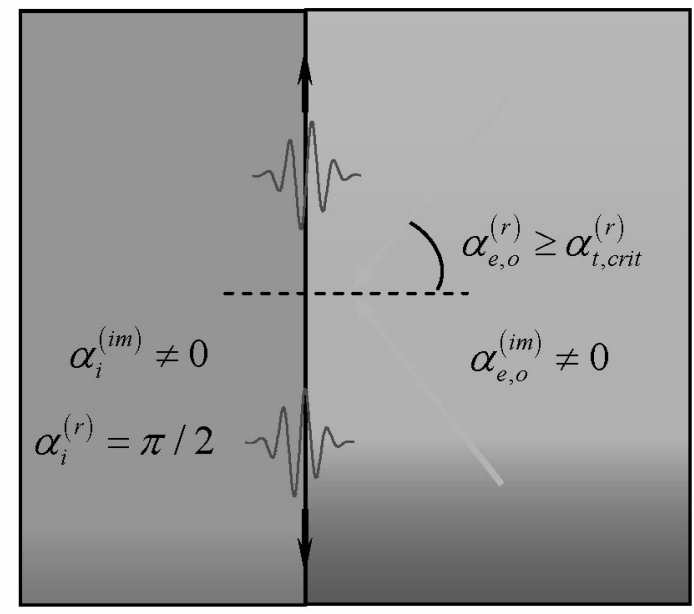

evanescent waves

Fig. 1. Illustration of how a nonparaxial beam radiated from a homogeneous medium $n_{1}<n_{o}, n_{e}$ induces two counter-propagating beams in an anisotropic medium (optically uniaxial crystal), and a set of evanescent waves.

A portion of homogeneous counter-propagating waves in the crystal with $\operatorname{Im}\left(k_{\perp}^{(o, e)}\right)=0$ has to be matched with a portion of evanescent waves in the isotropic medium. At the same time, all the evanescent waves in the crystal with $\operatorname{Im}\left(k_{\perp}^{(o, e)}\right) \neq 0$ have to be matched with the remaining portion of evanescent waves in the isotropic medium. As a consequence, the transmitted and reflected fields at the interface are strongly perturbed. The centre of gravity of the reflected field is shifted and the wave as a whole experiences a longitudinal and lateral shifts, too [15]. In our analysis, we will assume that the field inside the crystal at the interface has a well-defined and relatively simple form containing a regular vortex structure. However, the price for this simplicity is essential deformation of the reflected beam, the point remaining beyond our consideration. 


\section{Field structure of the paraxially annihilating vortex-beams}

Our starting point is to construct a simplest nonparaxial vortex-beam in the uniaxial crystal. In the work [20], we have already treated the basic principle of deriving the beam solutions to the Maxwell's equations in those crystals. Now we make use of the results obtained there and write out the electric and magnetic field components in the circularly polarised basis of the ordinary $\left(\left\{\mathbf{E}_{1}^{(o)}, \mathbf{H}_{1}^{(o)}\right\}\right)$ and extraordinary $\left(\left\{\mathbf{E}_{1}^{(e)}, \mathbf{H}_{1}^{(e)}\right\}\right)$ waves for the vortex-beam with the lowest order of topological charge $(l=\mp 1)$ in the input $z=0$ plane:

$$
\begin{aligned}
& E_{+, 1}^{(o)}=\left(\frac{v}{w_{0}}\right)\left(\frac{i z_{o}}{R_{o}}\right) \frac{j_{1}\left(k_{o} R_{o}\right)}{j_{1}\left(i k_{o} z_{o}\right)}, \\
& E_{-, 1}^{(o)}=-\left(\frac{u}{w_{0}}\right)\left(\frac{i z_{o}}{R_{o}}\right) \frac{j_{1}\left(k_{o} R_{o}\right)}{j_{1}\left(i k_{o} z_{o}\right)}, \\
& E_{z, 1}^{(o)}=0 \text {, } \\
& H_{+, 1}^{(o)}=-\left(\frac{v}{w_{0}}\right)\left(\frac{i n_{o} z_{o}}{R_{o}}\right)\left(\frac{z+i z_{o}}{R_{o}}\right) \frac{j_{2}\left(k_{o} R_{o}\right)}{j_{1}\left(i k_{o} z_{o}\right)}, \\
& H_{-, 1}^{(o)}=-\left(\frac{u}{w_{0}}\right)\left(\frac{i n_{o} z_{o}}{R_{o}}\right)\left(\frac{z+i z_{o}}{R_{o}}\right) \frac{j_{2}\left(k_{o} R_{o}\right)}{j_{1}\left(i k_{o} z_{o}\right)}, \\
& H_{z, 1}^{(o)}=-\left(\frac{i z_{o}}{w_{0}}\right)\left(\frac{1}{k_{o} R_{o}}\right)\left[2 j_{1}\left(k_{o} R_{o}\right)-\frac{k_{o} r^{2}}{R_{o}} j_{2}\left(k_{o} R_{o}\right)\right] \frac{1}{j_{1}\left(i k_{o} z_{o}\right)}, \\
& E_{+, 1}^{(e)}=\left(\frac{v}{w_{0}}\right)\left(\frac{i z_{e}}{R_{e}}\right)\left(\frac{\frac{n_{o}}{n_{3}} z+i z_{e}}{R_{e}}\right) \frac{j_{2}\left(k_{e} R_{e}\right)}{j_{2}\left(i k_{e} z_{e}\right)}, \\
& E_{-, 1}^{(e)}=\left(\frac{u}{w_{0}}\right)\left(\frac{i z_{e}}{R_{e}}\right)\left(\frac{\frac{n_{o}}{n_{3}} z+i z_{e}}{R_{e}}\right) \frac{j_{2}\left(k_{e} R_{e}\right)}{j_{2}\left(i k_{e} z_{e}\right)}, \\
& E_{z, 1}^{(e)}=\left(\frac{i n_{o}^{2} z_{e}}{n_{3}^{2} w_{0}}\right)\left(\frac{1}{k_{e} R_{e}}\right)\left[2 j_{1}\left(k_{e} R_{e}\right)-\frac{k_{e} r^{2}}{R_{e}} j_{2}\left(k_{e} R_{e}\right)\right] \frac{1}{j_{2}\left(i k_{e} z_{e}\right)}, \\
& H_{+, 1}^{(e)}=\frac{n_{o}^{2}}{n_{3}}\left(\frac{v}{w_{0}}\right)\left(\frac{i z_{e}}{R_{e}}\right) \frac{j_{1}\left(k_{e} R_{e}\right)}{j_{2}\left(i k_{e} z_{e}\right)}, \\
& H_{-, 1}^{(e)}=-\frac{n_{o}^{2}}{n_{3}}\left(\frac{u}{w_{0}}\right)\left(\frac{i z_{e}}{R_{e}}\right) \frac{j_{1}\left(k_{e} R_{e}\right)}{j_{2}\left(i k_{e} z_{e}\right)}, \\
& H_{z, 1}^{(e)}=0 \text {, }
\end{aligned}
$$


where the notation $u=x+i y, \quad v=x-i y, \quad r^{2}=x^{2}+y^{2}=u v, \quad R_{o}^{2}=u v+\left(z+i z_{o}\right)^{2}$, $R_{e}^{2}=u v+\left(\frac{n_{o}}{n_{3}} z+i z_{e}\right)^{2}$ is used, $j_{m}(x)$ is the spherical Bessel function of the first order [21], $m=0,1,2, \ldots, z_{o, e}=k_{o, e} w_{0}^{2} / 2, k_{o, e}=n_{o, 3} k_{0}, k_{0}$ stands for the wave number for the free space and $n_{o}, n_{3}$ are the refractive indices along the principal crystallographic axes.

At the same time, the mode beams

$$
\left\{\mathbf{E}_{2}^{(o)}, \mathbf{H}_{2}^{(o)}\right\}=\partial_{z}\left\{\mathbf{E}_{1}^{(o)}, \mathbf{H}_{1}^{(o)}\right\},\left\{\mathbf{E}_{2}^{(e)}, \mathbf{H}_{2}^{(e)}\right\}=\partial_{z}\left\{\mathbf{E}_{1}^{(e)}, \mathbf{H}_{1}^{(e)}\right\}
$$

obey also the Maxwell's equations. They take the following form:

$$
\begin{aligned}
& E_{+, 2}^{(o)}=\left(\frac{v}{w_{0}}\right)\left(\frac{i z_{o}}{R_{o}}\right)\left(\frac{z+i z_{o}}{R_{o}}\right) \frac{j_{2}\left(k_{o} R_{o}\right)}{j_{2}\left(i k_{o} z_{o}\right)}, \\
& E_{-, 2}^{(o)}=-\left(\frac{u}{w_{0}}\right)\left(\frac{i z_{o}}{R_{o}}\right)\left(\frac{z+i z_{o}}{R_{o}}\right) \frac{j_{2}\left(k_{o} R_{o}\right)}{j_{2}\left(i k_{o} z_{o}\right)}, \\
& E_{z, 2}^{(o)}=0 \text {, } \\
& H_{+, 2}^{(o)}=\left(\frac{v}{w_{0}}\right)\left(\frac{i n_{o} z_{o}}{R_{o}}\right)\left[\frac{j_{2}\left(k_{o} R_{o}\right)}{k_{o} R_{o}}-\left(\frac{z+i z_{o}}{R_{o}}\right)^{2} j_{3}\left(k_{o} R_{o}\right)\right] \frac{1}{j_{2}\left(i k_{o} z_{o}\right)}, \\
& H_{+, 2}^{(o)}=\left(\frac{u}{w_{0}}\right)\left(\frac{i n_{o} z_{o}}{R_{o}}\right)\left[\frac{j_{2}\left(k_{o} R_{o}\right)}{k_{o} R_{o}}-\left(\frac{z+i z_{o}}{R_{o}}\right)^{2} j_{3}\left(k_{o} R_{o}\right)\right] \frac{1}{j_{2}\left(i k_{o} z_{o}\right)}, \\
& H_{z, 2}^{(o)}=-\left(\frac{z+i z_{o}}{w_{0}}\right)\left(\frac{i n_{o} z_{o}}{R_{o}}\right)\left[2 \frac{j_{2}\left(k_{o} R_{o}\right)}{k_{o} R_{o}}-\frac{r^{2}}{R_{o}^{2}} j_{3}\left(k_{o} R_{o}\right)\right] \frac{1}{j_{2}\left(i k_{o} z_{o}\right)}, \\
& E_{+, 2}^{(e)}=\left(\frac{v}{w_{0}}\right)\left[\frac{j_{2}\left(k_{e} R_{e}\right)}{\left(k_{e} R_{e}\right)^{2}}-\frac{\left(\frac{n_{o}}{n_{3}} z+i z_{e}\right)^{2}}{R_{e}^{2}} \frac{j_{3}\left(k_{e} R_{e}\right)}{k_{e} R_{e}}\right] \frac{1}{N}, \\
& E_{-, 2}^{(e)}=\left(\frac{u}{w_{0}}\right)\left[\frac{j_{2}\left(k_{e} R_{e}\right)}{\left(k_{e} R_{e}\right)^{2}}-\frac{\left(\frac{n_{o}}{n_{3}} z+i z_{e}\right)^{2}}{R_{e}^{2}} \frac{j_{3}\left(k_{o} R_{o}\right)}{k_{e} R_{e}}\right] \frac{1}{N}, \\
& E_{z, 2}^{(e)}=-\frac{n_{o}^{2}}{n_{3}^{2}}\left[\frac{\left(\frac{n_{o}}{n_{3}} z+i z_{e}\right)}{w_{0} k_{e} R_{e}}\right]\left[2 \frac{j_{2}\left(k_{e} R_{e}\right)}{k_{e} R_{e}}-\frac{r^{2}}{R_{e}^{2}} j_{3}\left(k_{e} R_{e}\right)\right] \frac{1}{N},
\end{aligned}
$$




$$
\begin{aligned}
& H_{+, 2}^{(e)}=-\left(\frac{v}{w_{0}}\right) \frac{k_{o} n_{o}\left(\frac{n_{o}}{n_{e}} z+i z_{e}\right)}{\left(k_{e} R_{e}\right)^{2}} j_{2}\left(k_{e} R_{e}\right) \frac{1}{N}, \\
& H_{-, 2}^{(e)}=\left(\frac{u}{w_{0}}\right) \frac{k_{o} n_{o}\left(\frac{n_{o}}{n_{e}} z+i z_{e}\right)}{\left(k_{e} R_{e}\right)^{2}} j_{2}\left(k_{e} R_{e}\right) \frac{1}{N}, \\
& H_{z, 2}^{(e)}=0,
\end{aligned}
$$

where $N=\left[\frac{j_{2}\left(i k_{e} z_{e}\right)}{\left(i k_{e} z_{e}\right)^{2}}-\frac{j_{3}\left(i k_{e} z_{e}\right)}{i k_{e} z_{e}}\right]$.

Let us consider superpositions of the ordinary and extraordinary beams in Eqs. (4)(7),

$$
\begin{aligned}
& \left\{\mathbf{E}_{1}^{(o, e)}, \mathbf{H}_{1}^{(o, e)}\right\}=c_{1}\left\{\mathbf{E}_{1}^{(o)}, \mathbf{H}_{1}^{(o)}\right\}+c_{2}\left\{\mathbf{E}_{1}^{(e)}, \mathbf{H}_{1}^{(e)}\right\}, \\
& \left\{\mathbf{E}_{2}^{(o, e)}, \mathbf{H}_{2}^{(o, e)}\right\}=c_{3}\left\{\mathbf{E}_{2}^{(o)}, \mathbf{H}_{2}^{(o)}\right\}+c_{4}\left\{\mathbf{E}_{2}^{(e)}, \mathbf{H}_{2}^{(e)}\right\},
\end{aligned}
$$

where $c_{n}(n=1,2,3,4)$ are constant coefficients. As shown in [20], the beam fields given by Eqs. (4)-(7) turn into typical paraxial Laguerre-Gaussian beams of the lowest order in the paraxial limit $k_{o, e} z_{o, e}>>1$, so that one has

$$
\begin{aligned}
& \frac{z+i z_{o}}{R_{o}}, \frac{z \frac{n_{o}}{n_{3}}+i z_{e}}{R_{e}} \stackrel{k_{o, e} z_{o, e}>>1}{\longrightarrow} 1, \\
& j_{2 p}\left(k_{o, e} R_{o, e}\right) \stackrel{k_{o, e} z_{o, e}>>1}{\longrightarrow}-j_{2 p-2}\left(k_{o, e} R_{o, e}\right), \\
& j_{2 p+1}\left(k_{o, e} R_{o, e}\right) \stackrel{k_{o, e} z_{o, e}>1}{\longrightarrow}-j_{2 p-1}\left(k_{o, e} R_{o, e}\right), \\
& j_{1}\left(k_{o, e} R_{o, e}\right) \stackrel{k_{o, e} z_{o, e}>>1}{\longrightarrow} i j_{0}\left(k_{o, e} R_{o, e}\right), \\
& j_{2}\left(k_{o, e} R_{o, e}\right) \stackrel{k_{o, e} z_{o, e}>1}{\longrightarrow}-j_{0}\left(k_{o, e} R_{o, e}\right), \\
& \frac{1}{R_{o, e}} \stackrel{k_{o, e} z_{o, e}>>1}{\longrightarrow} \frac{1}{i z_{o, e} \sigma_{o, e}}, \sigma_{o, e}=1-i \frac{z}{z_{o, e}}, \\
& \frac{j_{0}\left(k_{o, e} R_{o, e}\right)}{j_{0}\left(i k_{o, e} z_{o, e}\right)} \stackrel{\exp \left(-\frac{r_{o, e}^{2}}{w_{0}^{2} \sigma_{o, e}>>1}\right)}{\longrightarrow} G_{o, e}=\frac{\sigma_{o, e}}{} .
\end{aligned}
$$

Our requirement is that the left-hand polarised components of the field superpositions (13) and (14) in the first and the second beam types should vanish at the plane $z=0$ in the paraxial approximation $\left(k_{o, e} z_{o, e}>1\right)$ : 


$$
\begin{gathered}
E_{-, 1}^{(o, e)}=c_{1} E_{-, 1}^{(o)}+c_{2} E_{-, 1}^{(e)} \stackrel{k_{o, e} z_{o, e} \gg>1}{\longrightarrow} 0, \\
E_{-, 2}^{(o, e)}=c_{3} E_{-, 2}^{(o)}+c_{4} E_{-, 2}^{(e)} \stackrel{k_{o, e} z_{o, e}>>1}{\longrightarrow} 0 .
\end{gathered}
$$

After tedious though simple mathematical transformations performed in Eqs. (13) and (14), we find that $c_{1}=c_{2}=c_{3}=c_{4}=1$ and

$$
\left\{\mathbf{E}_{1}^{(o, e)}, \mathbf{H}_{1}^{(o, e)}\right\} \stackrel{k_{o, e} z_{o, e}>1}{\longrightarrow}\left\{\mathbf{E}_{2}^{(o, e)}, \mathbf{H}_{2}^{(o, e)}\right\}
$$

i.e. the fields of the first and second beam types coincide with each other in the paraxial approximation.

Our second step is to construct the field

$$
\{\mathbf{E}, \mathbf{H}\}=\left\{\mathbf{E}_{1}^{(o, e)}, \mathbf{H}_{1}^{(o, e)}\right\}-\left\{\mathbf{E}_{2}^{(o, e)}, \mathbf{H}_{2}^{(o, e)}\right\} .
$$

The above nonparaxial field has a well-defined magnitude over the range $-\infty<z<\infty$, vanishing only in some limiting areas. However, it annihilates,

$$
\{\mathbf{E}, \mathbf{H}\} \stackrel{k_{o, e} z_{o, e} \gg 1}{\longrightarrow} 0,
$$

over all the range $-\infty<z<\infty$ in the paraxial limit $\left(k_{o, e} z_{o, e}>>1\right)$. It is worthwhile that the feature of paraxial annihilation is inherent in all the nonparaxial beams obtained as

$$
\left\{\mathbf{E}, \mathbf{H}_{n, m, p}=\frac{\partial^{m+n+p}}{\partial u^{m} \partial \nu^{n} \partial z^{p}}\left[N_{m, n, p}^{(1)}\left\{\mathbf{E}_{1}^{(o, e)}, \mathbf{H}_{1}^{(o, e)}\right\}-N_{m, n, p}^{(2)}\left\{\mathbf{E}_{2}^{(o, e)}, \mathbf{H}_{2}^{(o, e)}\right\}\right],\right.
$$

where $N_{m, n, p}^{(1,2)}$ are the corresponding normalising coefficients.

\section{Intrinsic features of the paraxially annihilating vortex-beams 4.1. Electric and magnetic fields}

What do the evanescent waves and the mismatching between the electric and magnetic fields contribute to annihilating process of the vortex-beams? To answer the question, let us consider the structure of the field components. The approximate boundary conditions given by Eqs. (22) and (23) provide minimisation of the left-hand polarised component of the nonparaxial beam in the input plane $z=0$ (in the paraxial approximation, this component vanishes), so that the right-hand polarised component contributes chiefly to the beam field at comparatively small crystal lengths $z$.

Fig. 2 illustrates the transverse and longitudinal distributions of the amplitudes and phases of the right-hand polarised components in the electric $\left(E_{+}\right)$and magnetic $\left(H_{+}\right)$ fields. A major striking feature is essential distinction in the structures of these fields. Both the electric and magnetic fields carry over negatively charged optical vortex centred at the beam axis. The vortex is encircled by a set of ring dislocations (or toroidal vortices) in the $z=0$ plane. However, in contrast to the partial mode beams in the field composition with dislocations positioned only in the $z=0$ plane, the components of the paraxially annihilating beam have ring dislocations distributed along the negative and positive $z$ directions. Despite the above resemblance, different positions and numbers of ring disloca- 
tions make the amplitude and phase structures of the electric and magnetic fields absolutely different. As the waist radius of the beam increases, the structures of these fields start to match, though their amplitudes tend quickly to zero. Total matching of the electric and magnetic fields causes annihilation of the beam.
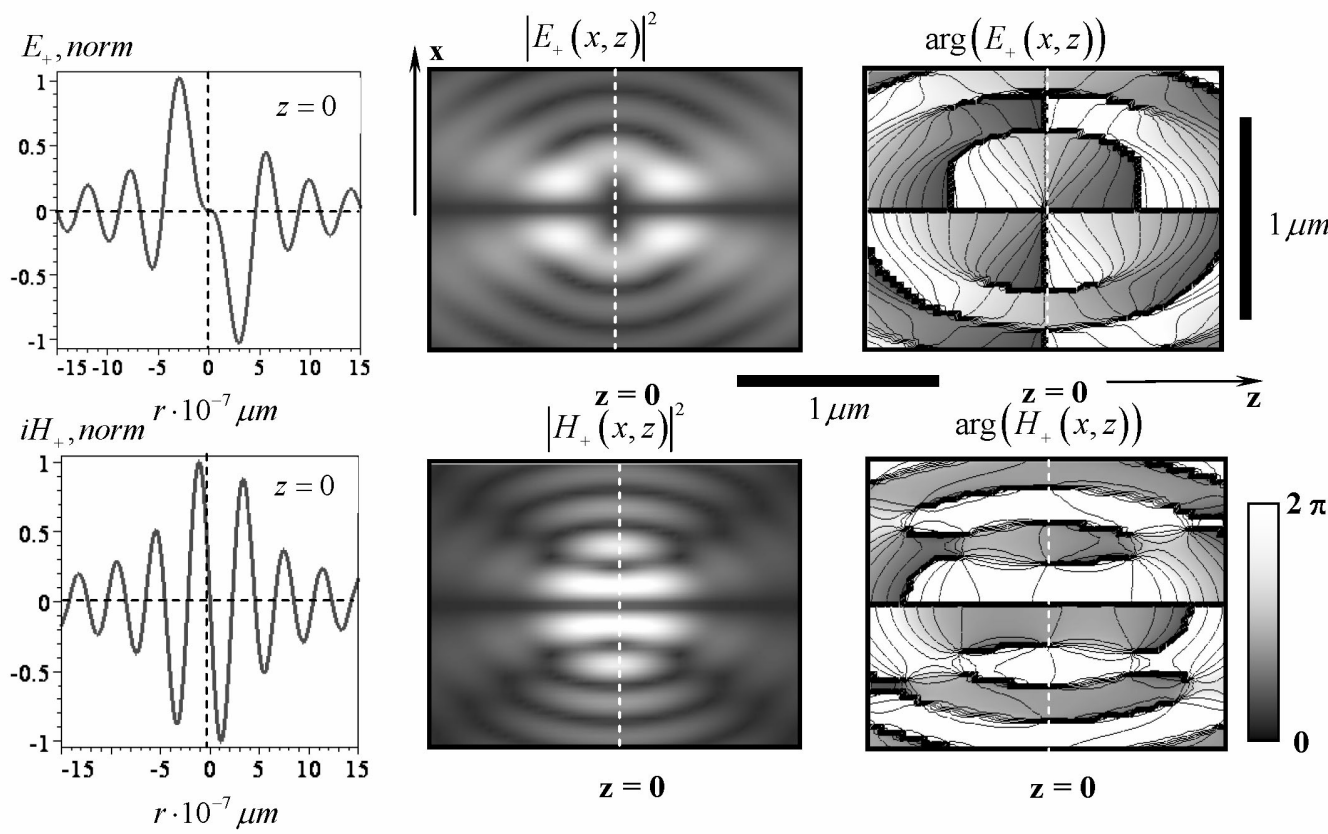

Fig. 2. Transverse and longitudinal distributions of right-hand polarised component in the electric $\left(E_{+}\right)$and magnetic $\left(H_{+}\right)$fields for the case of $w_{0}=0.1 \mu \mathrm{m}, n_{o}=2.3$ and $n_{3}=2.2$.

One of the constitutive points of our consideration is the impact of evanescent waves. The contributions of the evanescent and homogeneous waves to the field components may be estimated with Eqs. (2) and (3). The spectral distribution $\left(E_{+}, H_{+}\right)_{\text {hom }}=U_{\text {hom }}\left(k_{\perp}, \varphi^{\prime}\right)$ of the homogeneous wave,

$$
U_{\mathrm{hom}}\left(k_{\perp}, \varphi^{\prime}\right)=\pi e^{i m \varphi^{\prime}} \int_{0}^{\infty} d r \Psi(r, z=0) J_{m}\left(k_{\perp} r\right), \quad\left(0 \leq k_{\perp}<k_{o, e}\right),
$$

and the distribution $\left(E_{+}, H_{+}\right)_{\text {evan }}=U_{\text {evan }}\left(k_{\perp}, \varphi^{\prime}\right)$ of the evanescent one,

$$
U_{\mathrm{hom}}\left(k_{\perp}, \varphi^{\prime}\right)=\pi e^{i m \varphi^{\prime}} \int_{0}^{\infty} d r \Psi(r, z=0) J_{m}\left(k_{\perp} r\right), \quad\left(k_{o, e} \leq k_{\perp}<\infty\right),
$$

are defined by Eqs. (4a), (6a), (9a) and (11a) for the electric field component $\Psi=E_{+}$and by Eqs. (5a), (7a), (10a) and (12a) for the magnetic field component $\Psi=H_{+}$, along with Eq. (25). In the above relations, $\varphi^{\prime}$ means the azimuth angle in the $\mathbf{k}$ space. The integration limits $k_{o, e}$ imply that the spectral integrals given by Eqs. (28) and (29) are calculated separately for the ordinary and extraordinary mode beams. 
The curves shown in Fig. 3 describe spectral densities of the electric $\left(E_{+}\left(k_{\perp}\right)\right)$ and magnetic $\left(H_{+}\left(k_{\perp}\right)\right)$ fields components. Sharp peaks of the spectral curves occur at the transverse wave number $k_{\perp}^{(E)}=k_{o}$ for the electric field and at $k_{\perp}^{(H)}=k_{e}$ for the magnetic field. Any spectral density beyond the limits $k_{\perp}^{(E)}=k_{o}$ and $k_{\perp}^{(H)}=k_{e}$ refers to the evanescent waves. Different positions of these limits provide an extra evidence of drastic distinction between the spatial structures of the electric and magnetic fields in the beam. The profiles of the right-hand polarised components $\left|E_{+}(r)\right|$ and $\left|H_{+}(r)\right|$ shaped by homogeneous and evanescent waves are presented in Fig. 4. The contribution of evanescent waves to the paraxially annihilating beams seems to be relatively large in the nonparaxial case. The height of the spectral peaks in Fig. 3 quickly decreases with increasing $k_{o, e} z_{o, e}$ value. Besides, the evanescent waves do not contribute to the far field. However, in order to be sure of that fact, it is necessary to study the behaviour of the Poynting vector.

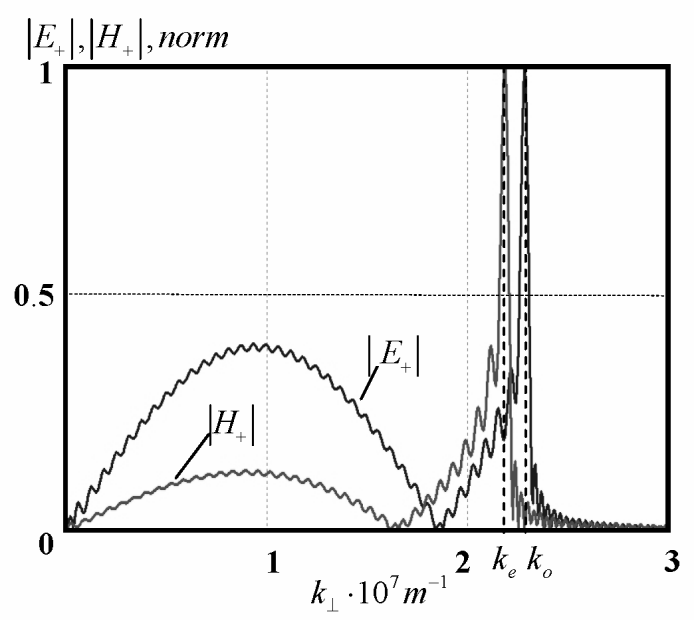

Fig. 3. Spectral distributions of electric $\left(E_{+}\left(k_{\perp}\right)\right)$ and $\left(H_{+}\left(k_{\perp}\right)\right)$ fields: $z=0$, $w_{0}=0.1 \mu \mathrm{m}$ and $n_{o}=2.3, n_{3}=2.2$.
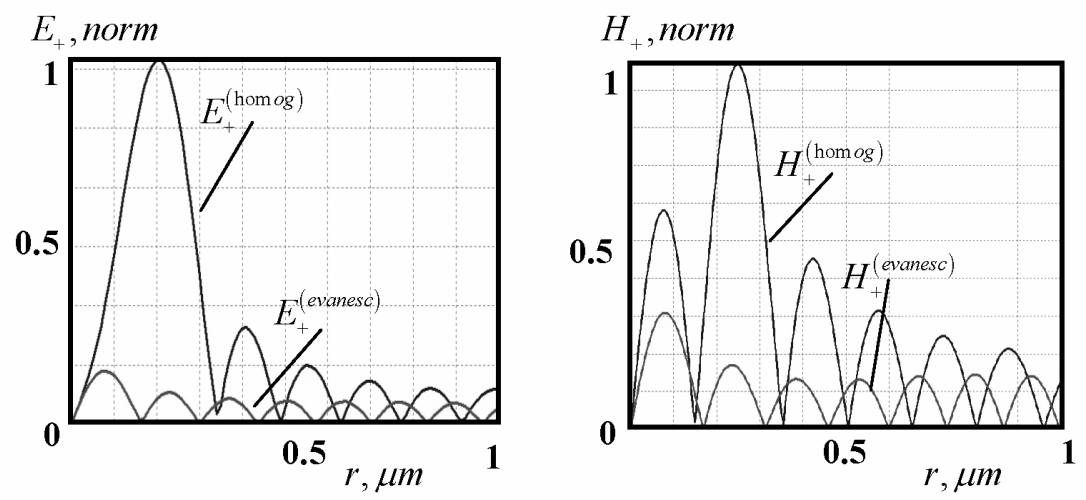

Fig. 4. Radial distributions of homogeneous and evanescent waves in the right-hand polarised components of nonparaxial electric $\left(E_{+}\right)$and magnetic $\left(H_{+}\right)$paraxially annihilating beam fields $\left(z=0, w_{0}=0.1 \mu \mathrm{m}\right.$, $n_{o}=2.3, n_{3}=2.2$ ) 


\subsection{Poynting vector}

The energy flux of the nonparaxial beam is described by the components of Poynting vector $\mathbf{P}$ (or the optical currents) $[14,22]$ that take into account the contributions of both the electric and magnetic fields:

$$
\begin{aligned}
\mathbf{P} & =\frac{c}{2 \pi} \operatorname{Re}\left(\mathbf{E} \times \mathbf{H}^{*}\right) \\
& =\frac{c}{2 \pi} \operatorname{Re}\left\{\mathbf{e}_{r}\left(E_{\varphi} H_{z}^{*}-E_{z} H_{\varphi}^{*}\right)+\mathbf{e}_{\varphi}\left(E_{z} H_{r}^{*}-E_{r} H_{z}^{*}\right)+\mathbf{e}_{z}\left(E_{r} H_{\varphi}^{*}-E_{\varphi} H_{r}^{*}\right)\right\},
\end{aligned}
$$

with $\left\{\mathbf{e}_{r}, \mathbf{e}_{\varphi}, \mathbf{e}_{z}\right\}$ being the unit vectors of the cylindrical basis. The curve $P_{z}(r, z=0)$ displayed in Fig. 5 characterises radial oscillations of the longitudinal energy flux in the paraxially annihilating beam. The zero of the $P_{z}$ component corresponds to polarisation singularities embedded in the beam. Due to oscillations, the component acquires both positive and negative values on the side lobes of the curve. This means that the optical currents form toroidal optical vortices in the $z=0$ plane.
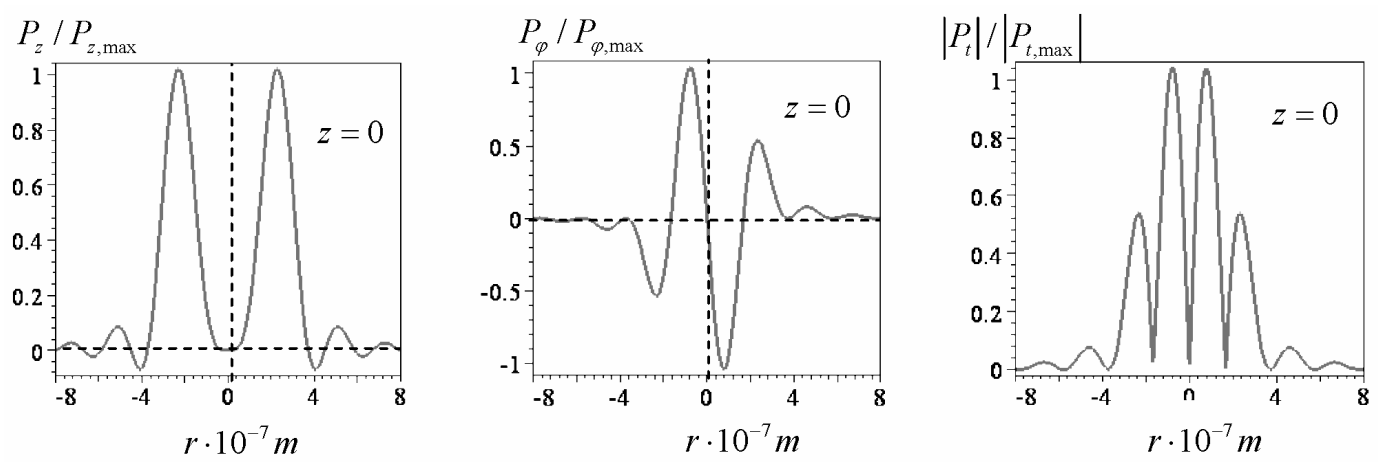

Fig. 5. Radial profiles of longitudinal $\left(P_{z}\right)$, azimuthal $\left(P_{\varphi}\right)$ and transverse $\left(P_{t}=\sqrt{P_{r}^{2}+P_{\varphi}^{2}}\right)$ components of the Poynting vector for the case of $n_{o}=2.3$, $n_{e}=2.2$ and $w_{0}=0.1 \mu \mathrm{m}$.

Fig. 6 represents a spatial evolution of the $P_{z}$ component, while Fig. 7 shows a detailed representation of the energy distribution between the components of the optical currents. There are two main peculiarities in the behaviour of the optical current. First, the portion of negative longitudinal components $P_{z}$ is sufficiently smaller than that of the corresponding positive values. The negative value of the $P_{z}$ component is also incommensurably smaller than that expected from the contributions of evanescent waves shown in Fig. 4. Although the evanescent waves play major part in shaping toroidal vortices in the $z$ plane, the contribution to the electric and magnetic fields is partially compensated in the optical currents. Second, the optical currents have additional focal planes in both the negative and positive areas of the $z$ axis. The density of the optical currents at first increases gradually with increasing $z$ coordinate (or decreasing $z$ coordinate, if $z<0$ ) and 
then immediately falls dawn beyond the additional focus. This effect clearly manifests itself in evolution of the curves shown in Fig. 6.

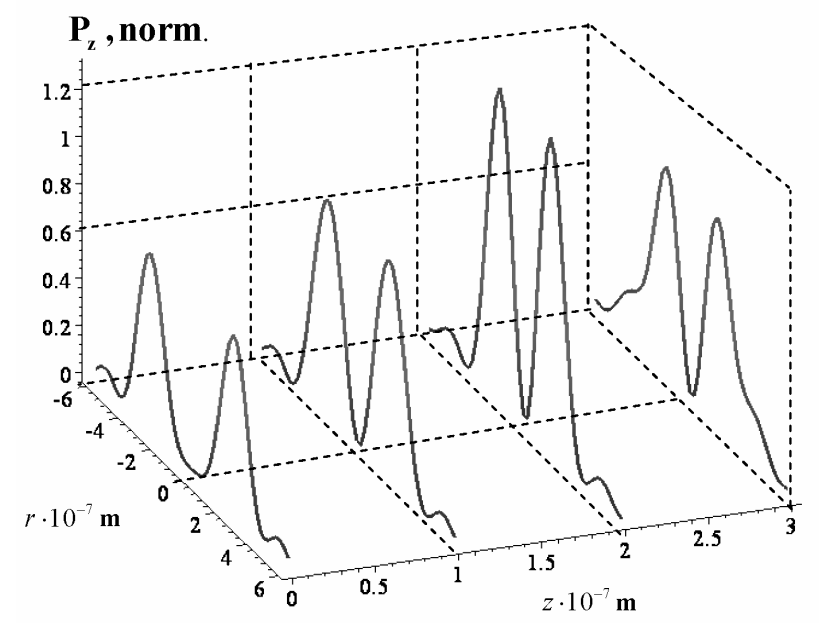

Fig. 6. Spatial evolution of normalised longitudinal component $P_{z}(r, z)$ at $w_{0}=0.1 \mu \mathrm{m}$.
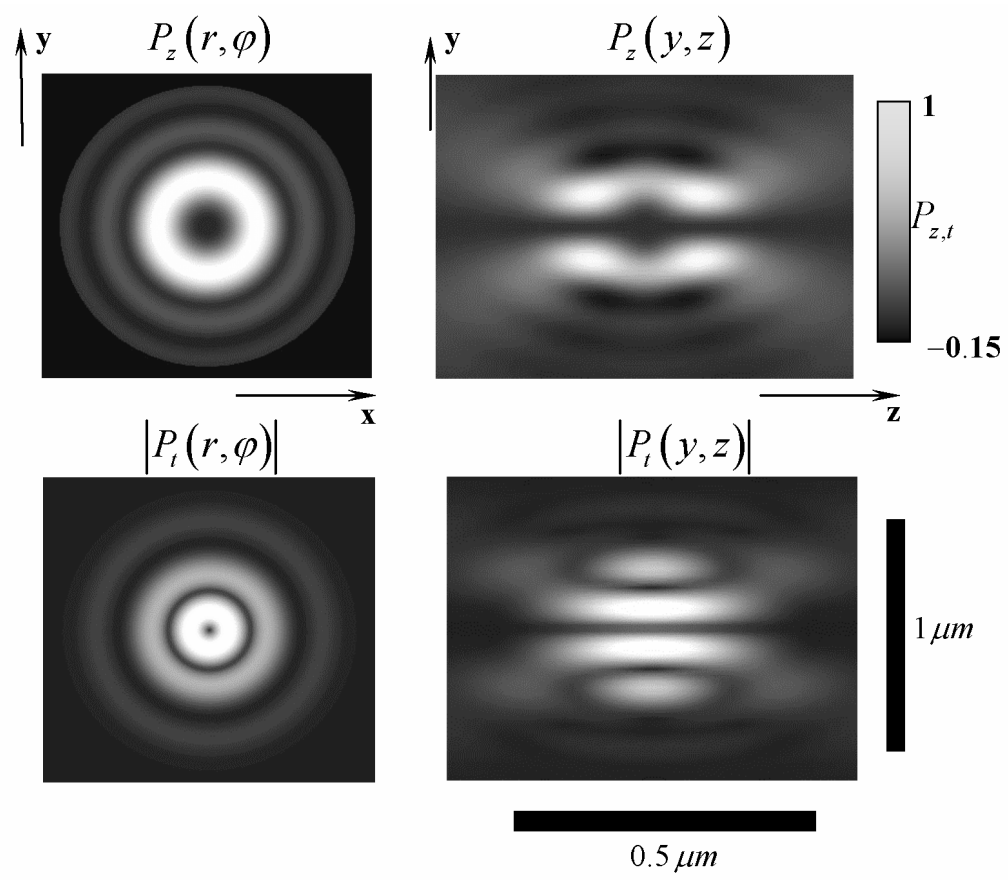

Fig. 7. Maps of optical current distributions in the paraxially annihilating beam $\left(w_{0}=0.1 \mu \mathrm{m}\right)$.

In order to trace transformation of the $P_{z}$ component along the $z$ axis and behaviour of the beam in the far field, we have plotted evolution of the $P_{z}^{(\max )}$ maximums versus 
the $z$ coordinate (see Fig. 8). For a comparison, we also illustrate evolution of the $P_{z, 1}^{(\max )}$ component of the partial beam $\left\{\mathbf{E}_{1}, \mathbf{H}_{1}\right\}$ included in the beam composition. Since the values of the optical current $P_{z, 1}^{(\max )}$ are significantly larger than $P_{z}^{(\max )}$, we have performed a normalization yielding the ratios of $P_{z, 1}^{(\max )}$ and $P_{z}^{(\max )}$ to their initial values at $z=0$. The curve $P_{z, 1}^{(\max )}(z)$ for the partial beam comes monotonically down and tends to zero as $1 / z$. The curve $P_{z}^{(\max )}$ has a peak in the plane of additional focus and then drops down in the same monotonic manner as $P_{z, 1}^{(\max )}(z)$. Thus, the field of the paraxially annihilating beam reveals the same evolution in the far field as that of typical nonparaxial beams. The additional focus is located in the vicinity of lateral toroidal optical vortices in the field components $E_{+}$and $H_{+}$(cf. with Fig. 2).

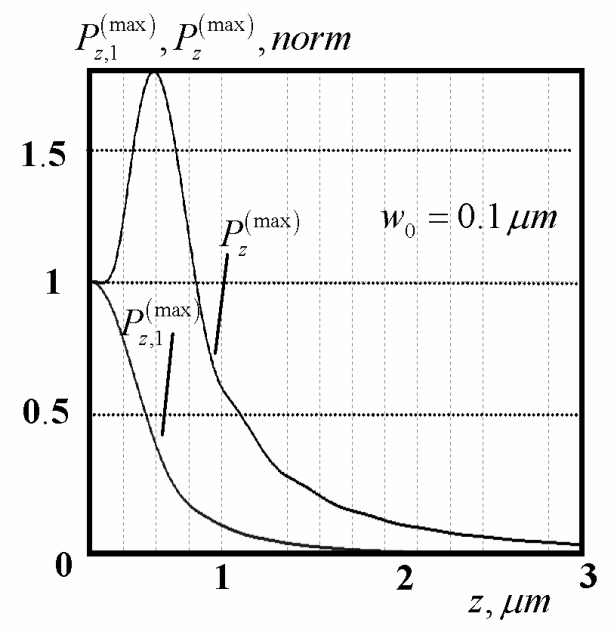

Fig. 8. Evolution of $P_{z}^{(\max )}$ parameter for the paraxially annihilating beam and $P_{z, 1}^{(\max )}$ for the partial beam $\left\{\mathbf{E}_{1}, \mathbf{H}_{1}\right\}$ along the crystal length.

A general pattern of the beam evolution may be described in terms of the optical currents given by Eq. (30) (see Fig. 9). The optical currents near the beam axis represent a uniform pattern with a slight deformation of the current lines. The deformation increases as the lines move away from the beam axis. The lines roll up into the loops and ovals in the vicinity of phase singularities of the field components, the loops being shaped not only in the $z=0$ plane (as it occurs for typical nonparaxial beams [20,23]) but also in the adjacent areas. With increasing beam radius $w_{0}$, the lateral loops and ovals approach to each other and annihilate in the $z=0$ plane. At the same time, the Poynting vector singularities [13] positioned in the $z=0$ plane, move away from the beam axis. When the beam radius increases up to $w_{0}=0.5 \mu \mathrm{m}$, the longitudinal energy flux $P_{z}$ becomes more than $10^{3}$ times smaller, when compare with that for the radius $w_{0}=0.1 \mu \mathrm{m}$. A further increase in the radius up to $w_{0}=2 \mu \mathrm{m}$ causes reduction of the flux by the factor of $10^{8}$, which is similar to annihilation of the beam. 


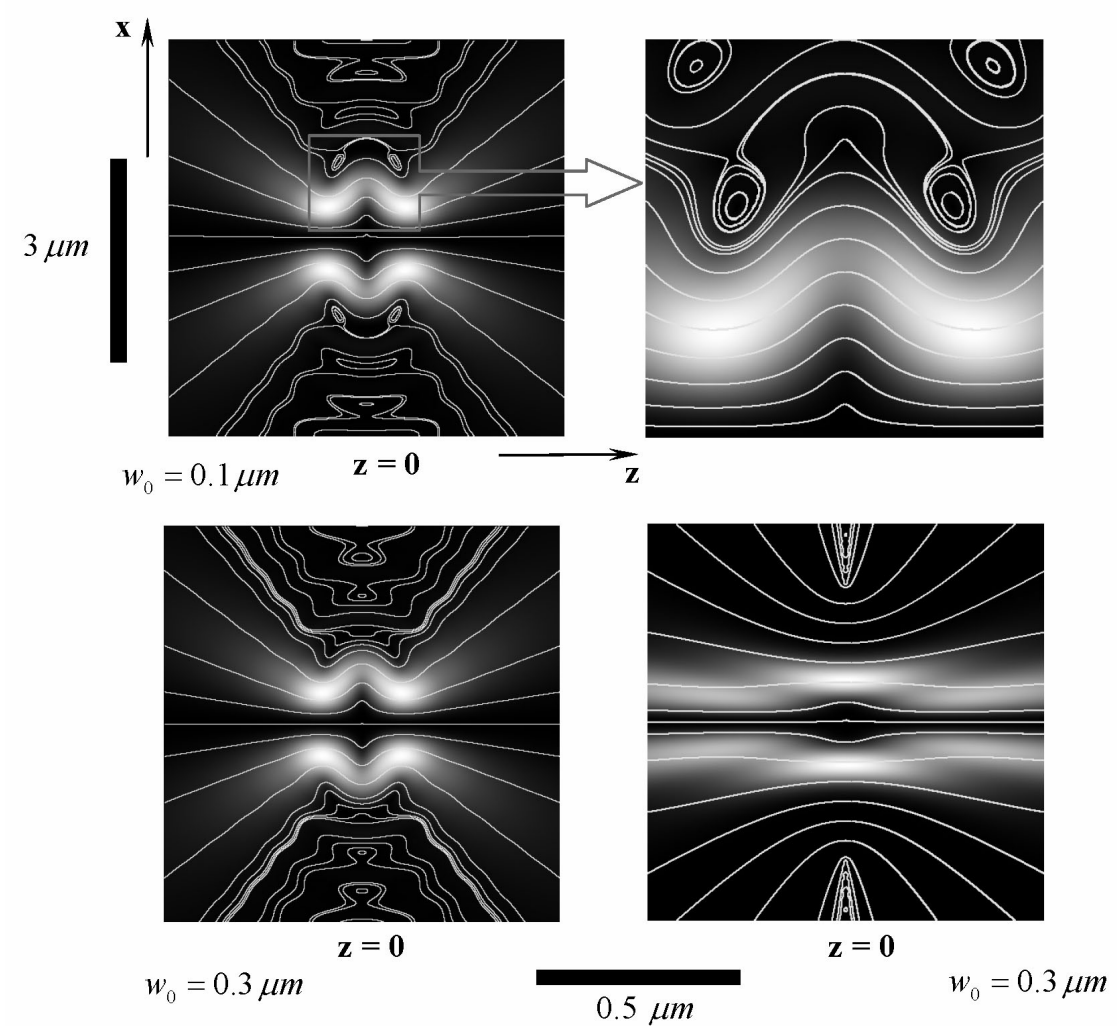

Fig. 9. Maps of optical currents in the paraxially annihilating beam for different beam waists $w_{0}$.

\section{Conclusions}

We have demonstrated that a unique property of the nonparaxial beams propagating along the optic axis of uniaxial crystals, the so-called paraxially annihilating beams, is to vanish when passing to the paraxial region. We have derived expressions for the fields obeying the Maxwell's equations and analysed them both in the terms of scalar phase singularities of the electric and magnetic field components and the optical current singularities. It is revealed that, besides of centred optical vortex, the components of the electric and magnetic fields have also a set of toroidal vortices (ring dislocations) positioned in the vicinity of $z=0$ plane rather than in the $z=0$ plane itself, as is the case with the typical nonparaxial beams. We have shown that the optical current lines in the paraxially annihilating beam form the loops and ovals near these phase singularities. When the waist radius increases, the lateral optical current singularities draw together and annihilate in the $z=0$ plane, while the singularities positioned in the $z=0$ plane move away from the beam axis. The optical current singularities in the $z=0$ plane are formed by the evanescent waves, while the lateral singularities originate from mismatching of the electric and magnetic fields in the nonparaxial beam. Complete matching of the electric and magnetic fields in the paraxial approximation results in disappearance of the paraxially annihilating beam. 
Although it is difficult to create experimentally non-truncated nonparaxial beams, we believe that further investigations of the nonparaxial beams in different optical media may enable experimental observations of novel unusual properties of these wave objects.

\section{Acknowledgement}

The authors thank K. A. Alexeyev for suggesting the term "paraxially annihilating beam".

\section{References}

1. Berry M V, 1994. Evanescent and real waves in quantum billiards and Gaussian beams. J. Phys. A: Math. Gen. 27: L391-L398.

2. Patanayak D N and Agraval G P, 1980. Representation of vector electromagnetic beams. Phys. Rev. A. 23: 1159-1164.

3. Felson L B, 1976. Evanescent waves. J. Opt. Soc. Am. 66: 751-760.

4. Kowarz M W, 1995. Homogeneous and evanescent contributions in scalar near-field diffraction. Appl. Opt. 34: 3055-3063.

5. Ruschin S and Leizer A, 1998. Evanescent Bessel beams. J. Opt. Soc. Am. A. 15: 1139-1143.

6. Berry M, 1998. Wave dislocation reactions in non-paraxial Gaussian beams. J. Mod. Opt. 45: $1845-1858$.

7. Wolf E and Folye J T, 1998. Do evanescent waves contribute to the far field? Opt. Lett. 23: 16-19.

8. Nye $\mathbf{J}$ and Hajnal J, 1987. The wave structure of monochromatic electromagnetic radiation. Proc. Roy. Soc. A. 409: 21-36.

9. Bialynicki-Birula I and Bialynicki-Birula Z, 2003. Vortex lines of the electromagnetic fields. Phys. Rev. A. 67: 062114.

10. Berry M, 2004. Riemann-Silberstein vortices for paraxial waves. J. Opt. A: Pure Appl. Opt. 6: S175-S177.

11. Mokhun I and Khrobatin R, 2008. Shift of application point of angular momentum in the area of elementary polarization singularity. J. Opt. A: Pure Appl. Opt. 10: 064015-064025.

12. Mokhun I, Brandel R and Viktorovskaya Ju, 2006. Angular momentum of electromagnetic field in areas of polarization singularities. Ukr. J. Phys. Opt. 7: 63-73.

13. Mokhun I, Mokhun A and Viktorovskaya Ju, 2006. Singularities of Poynting vector and the structure of optical fields. Ukr. J. Phys. Opt. 7: 129-141.

14. Berry M, 2009. Optical currents. J. Opt. A: Pure Appl. Opt. 11: 094001-09413.

15. Bonamo N and Depine R, 1997. Nonspecular reflection of ordinary and extraordinary beams in uniaxial media. J. Opt. Soc. Am. 14: 3402-3409

16. Torok P, Varga P, Lacazik L and Bonker G, 1995. Electromagnetic diffraction of light focused through a planar interface materials of mismatched refractive indices: an integral representation. J. Opt. Soc. Am. A. 12: 325-332.

17. Gasper J, Sherman G and Stamnest J, 1976. Reflection and refraction of an arbitrary electromagnetic wave at a planar interface. J. Opt. Soc. Am. 66: 955-961. 
18. Stamnes J and Dhayalan V, 2001. Transmission of two-dimensional Gaussian beam into a uniaxial crystal. J. Opt. Soc. Am. 18: 1662-1669.

19. Stamnest J and Sherman G, 1978. Radiation of electromagnetic fields in biaxially anisotropic media. J. Opt. Soc. Am. 68: 502-508.

20. Fadeyeva T, 2010. Nonparaxial wave beams in a uniaxial birefringent crystal. Ukr. J. Phys. Opt. 11: 44-60.

21. Abramowitz M and Stegun I A. Handbook of mathematical functions. $10^{\text {th }}$ ed., NBS (1972).

22. Born M and Wolf E. Principles of optics. New York: Cambridge University Press (1999).

23. Volyar A, Shvedov V and Fadeyeva T, 2001. Structure of nonparaxial Gaussian beams near the focus: 2. Optical vortices. Opt. Spectr. 90: 104-112.

Fadeyeva T., Valkov I. and Volyar A., 2010. Paraxially annihilating vortex-beams in uniaxial crystals. Ukr.J.Phys.Opt. 11: 74-89.

Анотація. Розглянуто специфічні властивості параксіально анігілюючих пучків. Одержано вирази для пучків иьього типу, які задовольняють рівнянням Максвела. Виявлено, щзо походження цих пучків пов'язано з неузгодженням електричного та магнітного полів, а також із швидко затухаючими хвилями в компонентах поля. Повне узгодження електричного та магнітного полів в параксіальному наближенні зумовлює зникнення параксіально анігілюючих пучків. 